\title{
Zonal based Deterministic Energy Efficient Clustering Protocol for WSNs
}

\author{
Prabhleen Kaur \\ Punjab Institute of Technology, Kapurthala \\ (PTU Main Campus), Punjab \\ India
}

\author{
Rajdeep Singh \\ Punjab Technical University \\ Jalandhar, Punjab \\ India
}

\begin{abstract}
Wireless Sensor Network has gained a tremendous attention of researchers due to its vast applications. Wireless sensor network consists of small size and low powered smart sensor nodes deployed in the region to be monitored. Sensor nodes sense the data and send the sensed data to sink directly or by coordinating with other sensor nodes. It is difficult to change the network topology and battery of sensor nodes. Hence, a key point to increase stability period and network lifetime is to efficiently utilize the energy of sensors. In this paper, we propose a new protocol, zonal based deterministic energy efficient clustering protocol (ZDEC) in which sensor field is divided into zones. Cluster heads are elected based on residual energy such that each zone contains minimum one cluster head. Our proposed protocol ensures uniform distribution of cluster heads which leads to the uniform energy dissipation over the sensor field. Simulations show that our proposed protocol increase stability period and network lifetime when compared with LEACH, ESEP and DEC in heterogeneous environment significantly.
\end{abstract}

\section{Keywords}

Wireless Sensor Networks, Cluster, Zone, Uniform, Deterministic

\section{INTRODUCTION}

WSNs are considered as one of the best source for various monitoring applications which are out of range from human perspective. WSNs consist of large number of small size sensor nodes for monitoring of specified parameters. Sensor nodes sense the data and send the sensed data to base station (BS) directly or by coordinating with other sensor nodes. Once the network is deployed, it is impossible to change the network topology and battery of sensor nodes. Hence suitable protocols and applications should be developed for balanced energy dissipation among sensor nodes to increase stability period and network lifetime. On the basis of initial energy equipped in sensor nodes, protocols can be divided into two protocols - homogeneous and heterogeneous protocols. In homogeneous protocols, all the sensor nodes are equipped with same amount of initial energy and on the other hand, in heterogeneous protocols the sensor nodes are equipped with different levels of initial energy. LEACH [3] and TEEN [5] and are the examples of homogeneous protocols. SEP [6], ESEP [1], DEC [2] and DDEC [7] are examples of heterogeneous protocols. Numbers of different routing protocols are proposed by different authors described in [1], [2], [4], [6] and [7] to increase stability period and network lifetime using different approaches. Clustering approach performs better than direct transmission and multi-hop approach. In direct transmission, nodes send the sensed data directly to sink and thus the nodes farther from sink consume more energy and hence degrade the performance of network. In multi-hop each sensor node sends the sensed data to other sensor node towards sink and hence it performs better than direct transmission by short distance data transmission mechanism. But due to more load on the sensor nodes that are near to the base station, degrades the performance. In clustering approach, clusters are formed such that each cluster has a cluster head $(\mathrm{CH})$ and cluster members $(\mathrm{CMs})$. Cluster members send the sensed data to respective $\mathrm{CH}$. Each Cluster head aggregates the data received from cluster members and send aggregated data to sink. We propose a new protocol ZDEC which ensures balanced and uniformly distributed election of cluster heads over the sensor field. And hence it ensures uniform dissipation of energy of sensor nodes which increases the stability period and network lifetime significantly.

\section{RELATED WORK}

\subsection{Low Energy Adaptive Clustering Hierarchy Protocol (LEACH)}

It is called a dynamic clustered approach as described in [3]. It gives each node an equal chance to become cluster head. It consists of two phases- setup phase and steady phase. In setup phase, cluster heads are elected probabilistically. Each node is assigned a random number between 0 and 1 . A node becomes a cluster head if the assigned random number is less than a threshold. In steady phase, data transmission takes place.

\subsection{Enhanced Stable Election Protocol (ESEP)}

It is an extension of stable election protocol [6] described in [1] having three types of nodes in terms of energy: advanced nodes, intermediate nodes and normal nodes. Advanced nodes are a proportion of nodes $m$ out of total number of nodes $n$ such that their energy is $\alpha$ times more than normal nodes. Intermediate nodes are a proportion of nodes $b$ out of total nodes $\mathrm{n}$ such that each node having energy $\mu$ times greater than normal nodes. The energy of intermediate nodes is between normal and advanced nodes. Rest of total nodes $\mathrm{n}$ are normal nodes having energy less than both intermediate and advanced nodes. Cluster heads are elected based on the energy levels of nodes. Therefore in ESEP, energy dissipation is controlled according to three types of nodes.

\subsection{Deterministic Energy Efficient Clustering Protocol (DEC)}

It is a clustering protocol described in [2]. It guarantees the election of fixed number of cluster heads in each round. It elects cluster head based on residual energy of sensor nodes solely. In setup phase, at round $\mathrm{r}=\mathrm{m}$, base station elects $\mathrm{N}_{\mathrm{opt}}$ cluster heads over the sensor field (where $\mathrm{N}_{\mathrm{opt}}$ is optimal number of $\mathrm{CHs}$ to be elected and is given as inverse of optimal probability $\mathrm{P}_{\mathrm{opt}}$ ). Base station takes part in election of cluster head only when $r=1$. Cluster heads advertise (ADV) their role using CSMA/MAC protocol. Cluster members send the join-request message to cluster head from which they receive maximum signal strength. Join-request contains $\mathrm{CH}$ - 
ID, CM-ID and CM-RE. In this way, cluster head comes to know the residual energy of all its cluster members and cluster head uses this information to make cluster head for next round. Before the start of steady phase, $\mathrm{CH}$ elects the high residual energy node in its cluster as the cluster head for next round. $\mathrm{CH}$ sends a TDMA schedule to all of its cluster members. In steady phase, CMs send the sensed data in its time slot allotted by cluster head without collision. $\mathrm{CH}$ receives the data from all its cluster members and aggregates the received data. $\mathrm{CH}$ sends the aggregated data to sink. It ends the current round but since the cluster heads for next round are already chosen in this round, DEC decreases the overhead.

Since DEC elects $\mathrm{CH}$ based on residual energy only, hence sometimes more number of a cluster heads accumulate at one side as shown in Fig 1.

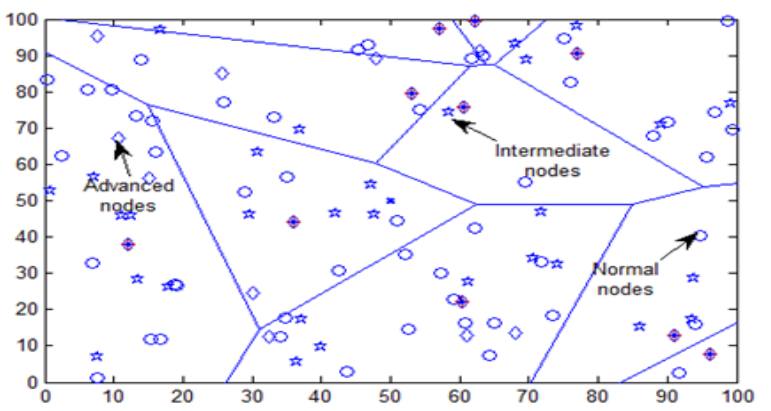

Fig 1: Non uniform distribution of cluster heads in DEC

Hence the cluster members farther from its cluster head have to spend more energy to send sensed data to cluster head [8]. Thus DEC does not ensure balance energy dissipation throughout the network. We propose an enhancement of DEC protocol called zonal deterministic energy efficient clustering protocol (ZDEC) in which network is portioned into zones and ensures uniform distribution of cluster head which leads to the uniform energy dissipation over the field.

\section{ENERGY MODEL}

We consider the energy and data aggregation model as described in [2]. Energy consumption for communication is far greater than energy consumption for sensing and processing. Hence for simplicity, we consider only the energy consumption for communication. According to the network model illustrate in Fig. 2, in order to transmit an L-bit message over distance $d$, the energy dissipated by radio is given as following:

$$
\mathrm{E}_{\mathrm{TX}}(\mathrm{L}, \mathrm{d})=\left\{\begin{array}{c}
\mathrm{LE}_{\text {elec }}+\mathrm{L} \varepsilon_{\mathrm{fs}} \mathrm{d}^{2}, \mathrm{~d}<\mathrm{d}_{0} \\
\mathrm{LE}_{\text {elec }}+\mathrm{L} \varepsilon_{\mathrm{amp}} \mathrm{d}^{4}, \mathrm{~d} \geq \mathrm{d}_{0}
\end{array}\right.
$$

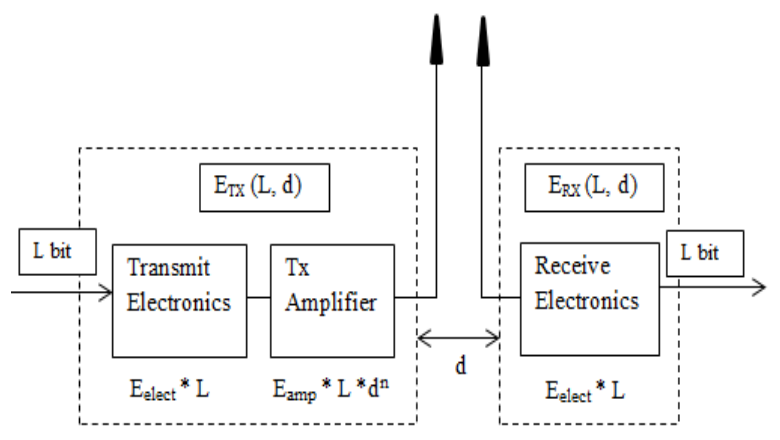

Fig 2: Network Model
And to receive message per bit, energy dissipated by radio is given as following

$$
\mathrm{E}_{\mathrm{RX}}(\mathrm{L})=\mathrm{LE}_{\text {elec }}
$$

And to aggregate $m$ such type of messages, the energy consumption is given as following

$$
\mathrm{E}_{\mathrm{DX}}=\mathrm{mLE}_{\mathrm{DA}}
$$

In equations (1) and (2), $\mathrm{E}_{\text {elec }}$ denotes the energy consumption to transmit or receives per bit. In (3), $\mathrm{E}_{\mathrm{DA}}$ denotes the energy consumption for aggregating 1 bit message. To transmit the data over a distance (d) with an acceptable SNR, the amplification energy $(\varepsilon)$ is expended to overcome either the free space (fs) or multi-path (amp) loss. The choice of $\varepsilon f s$ and $\varepsilon_{\text {amp }}$ depends on the transmission distance d. If $\mathrm{d}<\mathrm{d}_{0}$ then free space channel factor is used and if $\mathrm{d}>\mathrm{d} 0$ then multipath fading factor is used. By equating two cases of equation (1), $d_{0}$ is given as, $\mathrm{d}_{0}=\sqrt{ }\left(\varepsilon_{\mathrm{fs}} / \varepsilon_{\mathrm{amp}}\right)$

We also assume a symmetric radio channel, i.e. the same amount of energy is required to transmit L-bit message from node A to B and vice versa. Also in the previous studies such as in LEACH and the likes, they assumed a perfect data aggregation in which packets received from cluster members are aggregated by their respective cluster heads and only a single packet is forwarded to the BS. A similar model is assumed in this study.

\section{PROPOSED PROTOCOL}

In this section, we describe our proposed protocol ZDEC. We deploy random nodes in $100 \mathrm{~m} \times 100 \mathrm{~m}$ field. BS collects information regarding location of all sensor nodes in the network. BS virtually divides the network into 8 zones (Zone1, Zone2, zone3, zone4, zone5, zone6, zone7, Zone8) depending on graphical layout of network as shown in fig. 3 . ZDEC ensures the election of cluster head with minimum of $\mathrm{N}_{\text {opt }} / 8$ number of cluster heads in each zone. Doing such sort of partitioning and distributing cluster head in each zone ensures uniform and well distribution of $\mathrm{CHs}$ throughout layout of network. Hence it yields efficient energy utilization of sensor nodes.

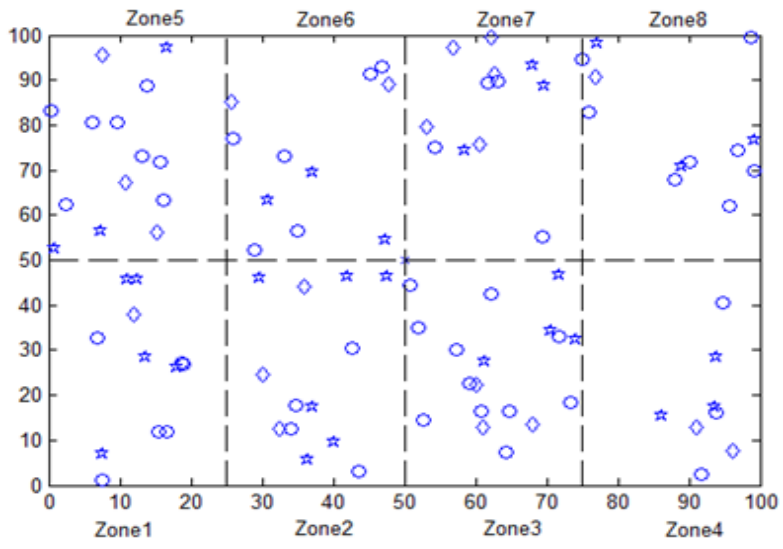

Fig 3: Partition of network into 8 virtual zones

ZDEC consists of two phases: Setup Phase and Steady-state Phase.

We modify the setup phase of DEC but keep the steady-state phase same. Since node's energy and node's zone determined a priori, the $\mathrm{CH}$ election process is reorganized using residual energy and zone id of each node. In setup phase cluster establishment takes place. ZDEC elects cluster heads based on 
residual energy such that each zone has minimum $\mathrm{N}_{\text {opt }} / 8$ cluster heads. At round $\mathrm{m}$, BS elects $\mathrm{N}_{\text {opt }}$ cluster heads for network such that each zone has minimum $\mathrm{N}_{\text {opt }} / 8$ cluster heads. BS takes part in election of cluster head only when $r=$ 1. The elected cluster heads advertise their roles using CSMA/MAC protocol just as in DEC. Sensor nodes send joinrequest message to cluster head from which they receive maximum signal strength. Clusters thus formed can cover more than one zone as depicted in figure 4. However, in ZDEC unlike in DEC, join-request message contains CH-ID, CM-ID, CM-RE and CM-ZONE_ID. In this way RE (residual energy) and ZONE_ID of cluster members are received by their respective cluster heads. This information is used for election of cluster heads for subsequent round and thus localized. After the setup phase, steady phase starts, but before the completion of this phase, $\mathrm{CH}$ checks the piggybacked CM-RE's and CM-ZONE_ID's information received to elect the new cluster head for next round. $\mathrm{CH}$ elects the highest residual energy node including itself in its cluster as 'candidate cluster head' for the next round and compares its ZONE_ID. It makes the 'candidate cluster head' as a 'confirm cluster head' for next round if condition for desired number of $\mathrm{CHs}$ in that specific zone is not met otherwise it chooses next high residual energy node in its cluster as a 'new candidate cluster head'. Similarly the same process continues for each 'candidate cluster head' checking for rest of the zone covered by cluster.

Once minimum $\mathrm{N}_{\mathrm{opt}} / 8$ 'confirm cluster heads' are elected in each zone for next round, remaining cluster heads are elected same as DEC. Once the new CHs for next round are decided, all the data from current round is transmitted to the BS. The current round $(\mathrm{r}=\mathrm{m})$ ends (a perfect synchronization is assumed, just as in DEC). The next round $r=m+1$ begins; but since the new CH's are elected already in the previous round which are also well distributed over the sensor field, they broadcast their roles in the new round. CMs join their cluster as explained above. This process continues till the last node dead. With this approach energy of sensor nodes is utilized efficiently and also balances the energy dissipation over the sensor field. Fig 4 shows the uniform distribution of cluster heads over the sensor field. The zones are bounded by dashed lines and clusters are bounded by solid line.

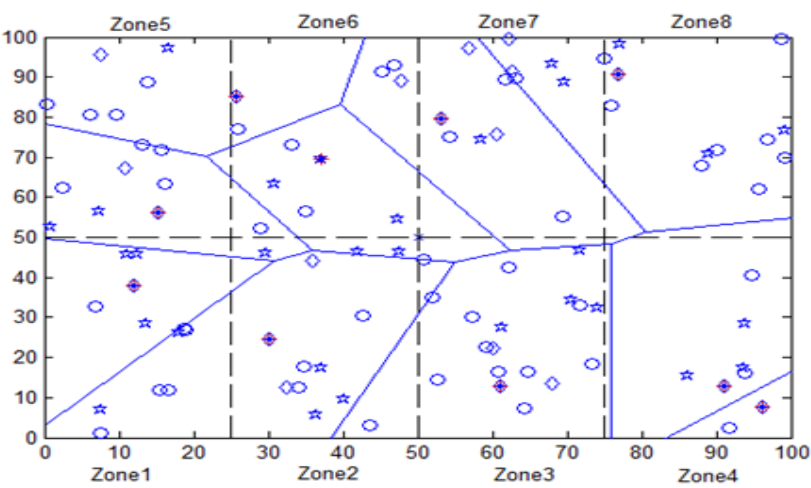

Fig 4: Uniform distribution of cluster heads in ZDEC with minimum $\mathbf{N}_{\text {opt }} / 8$ cluster heads in each zone

\section{SIMULATIONS}

To compare the performance of ZDEC, we perform simulations using MATLAB. We evaluate and compare performance of ZDEC with LEACH, ESEP and DEC protocols in heterogeneous environment.

\section{Performance Metrics}

- Stability period: The interval from start of network till the first node dies (FND). This period is also called stable region.

- Instability period: The period from first node die till the last node dies (LND).

- $\quad$ PNA: The interval from first node dies till the 90 percent node alive.

- Number of alive nodes per round

- Number of dead nodes per round

- Throughput: Number of packets sent from cluster heads to sink.

Table 1: Parameter Settings

\begin{tabular}{|c|c|}
\hline Parameter & Values \\
\hline $\mathrm{E}_{\text {elec }}$ & $50 \mathrm{nj} / \mathrm{bit}$ \\
\hline $\mathrm{E}_{\mathrm{DA}}$ & $50 \mathrm{nj} / \mathrm{bit} / \mathrm{message}$ \\
\hline $\mathrm{E}_{0}$ & $0.5 \mathrm{~J}$ \\
\hline $\mathrm{L}$ & 4000 \\
\hline $\mathrm{P}_{\mathrm{opt}}$ & 0.1 \\
\hline$\varepsilon_{\mathrm{fs}}$ & $10 \mathrm{pJ} / \mathrm{bit} / \mathrm{m}^{2}$ \\
\hline$\varepsilon_{\mathrm{amp}}$ & $0.0013 \mathrm{pJ} / \mathrm{bit} / \mathrm{m}^{4}$ \\
\hline $\mathrm{n}$ (no. of nodes) & 100 \\
\hline
\end{tabular}

A network consists of 100 nodes having three types of nodes with variation in initial energy. We have considered a squared sensor field with dimensions $100 \mathrm{~m} \times 100 \mathrm{~m}$. All the three types of nodes are randomly and uniformly distributed over the sensor field. The energy of sensor nodes varied between $0.5 \mathrm{~J}$ to $2.25 \mathrm{~J}$ and the location of BS is considered at the center of field. For the sake of complete fairness and brevity, the total energy of system for each protocol is considered same. In each LEACH, ESEP, DEC and ZDEC protocol, 20\% nodes are equipped with $2 \mathrm{~J}$ of energy, $30 \%$ nodes with $1.25 \mathrm{~J}$ of energy and $50 \%$ nodes with $0.5 \mathrm{~J}$ of energy. Hence the total energy of system for each protocol is $102.5 \mathrm{~J}$ as taken in [2]. Common parameters used in our simulations are shown in Table 1.

Fig 5 shows the rate of nodes in network which are alive and Fig 6 shows rate of nodes in network which are dead with respect to number of rounds. In these results, it is clear that the stability period of LEACH is least among all the protocols as it elects cluster heads probabilistically. Also it is expected as it is designed for homogeneous network. ESEP has more stability period than LEACH as ESEP elects cluster heads using different threshold using different probabilities for three different types of nodes varied in initial energy. But since ESEP elects cluster heads probabilistically, it has less stability period than DEC and ZDEC. DEC has more stability period than LEACH and ESEP because it elects cluster heads 
deterministically and cluster heads are elected on the basis of residual energy of nodes.

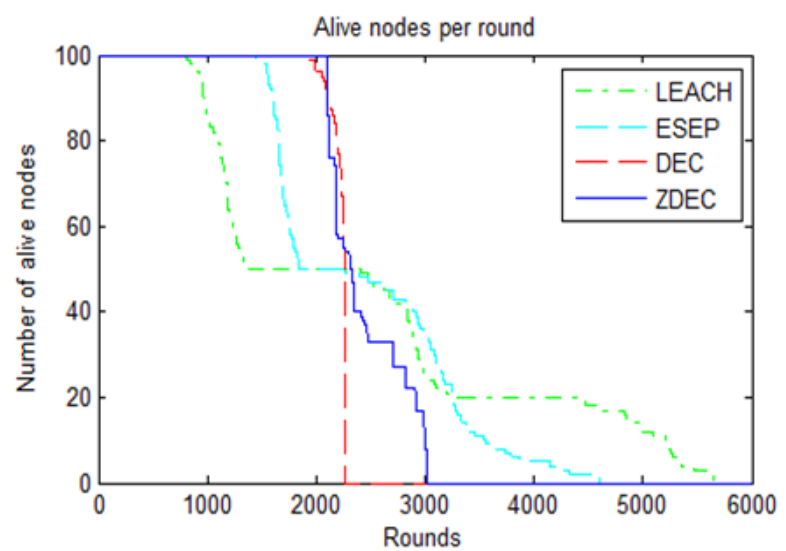

Fig 5: Alive nodes per round

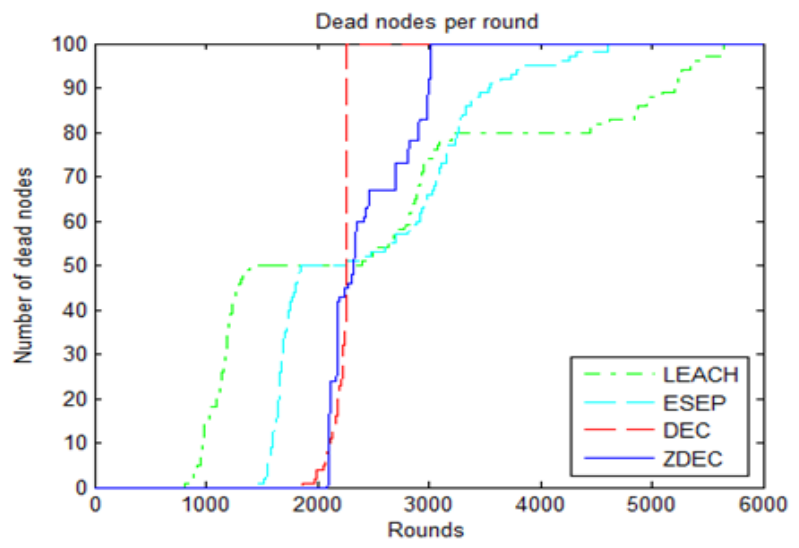

Fig 6: Dead nodes per round

ZDEC has highest stability period than all other protocols discussed above. It elects cluster heads uniformly distributed over the sensor field and hence energy dissipation is also uniform. Therefore it increases the stability period. Also in ZDEC, cluster heads are elected based on residual energy deterministically. ZDEC has stability period greater than LEACH and ESEP by a factor of 2.4 and 1.4 respectively. Also, the stability period and network lifetime of ZDEC is greater than DEC by $12.7 \%$ and $22 \%$ respectively.

The comparison based on first node die (FND), PNA (90, percent of nodes alive) and network lifetime (Last node die, LND) is shown in Table 2.

Table 2: Average of network lifetime of the sensors for 25 trials

\begin{tabular}{|l|l|l|l|}
\hline Protocols & FND & PNA & LND \\
\hline LEACH & 840 & 1100 & 4995 \\
\hline ESEP & 1450 & 1630 & 3751 \\
\hline DEC & 1849 & 2100 & 2350 \\
\hline ZDEC & 2095 & 2110 & 3000 \\
\hline
\end{tabular}

It is depicted from Table 2 that ZDEC has highest stability period (FND) than all the other protocols. Though the network lifetime of LEACH and ESEP is more than ZDEC but ZDEC is best suited for those applications which require $90-100 \%$ monitoring capability to get reliable data.

Fig 7 shows the comparison of ZDEC with LEACH, ESEP and DEC in terms of throughput per round with same parameters as discussed above. Throughput is total number of packets send from $\mathrm{CHs}$ to BS in whole network lifetime.

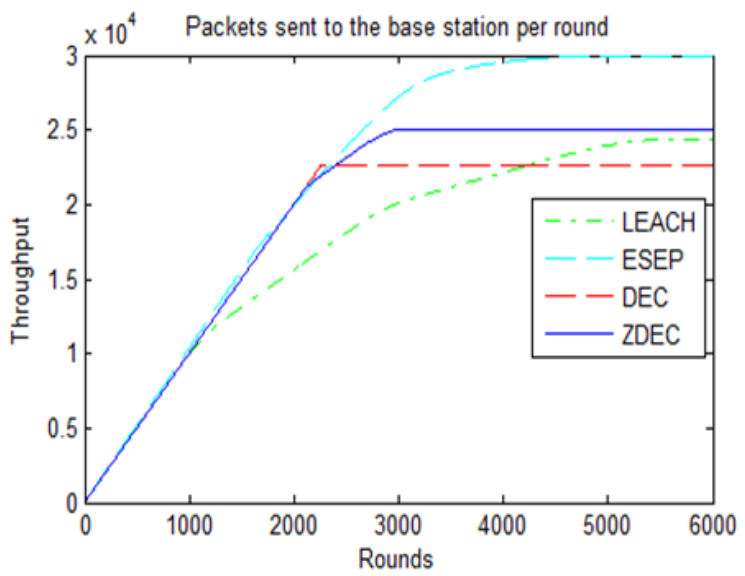

Fig 7: Packets sent to base station per round

From the above results, it is depicted that ESEP has highest throughput than all other protocols. Its throughput keeps on increasing and reaches $3 \mathrm{kbps}$ and then become constant after 4000 rounds. Whereas, LEACH has $2.4 \mathrm{kbps}$ throughput which is 22\% less than ESEP however its throughput is higher than DEC. DEC has $2.2 \mathrm{kbps}$ throughput which is $32 \%$ less than ESEP and $7.1 \%$ less than LEACH. Our proposed protocol ZDEC has throughput $2.5 \mathrm{kbps}$. The throughput of ZDEC is greater than DEC and LEACH by $10.6 \%$ and $2.1 \%$ respectively.

In Fig 8, there is a comparison of LEACH, ESEP, DEC and ZDEC with same parameters in terms of energy consumption per round. ZDEC flattens energy consumption than DEC.

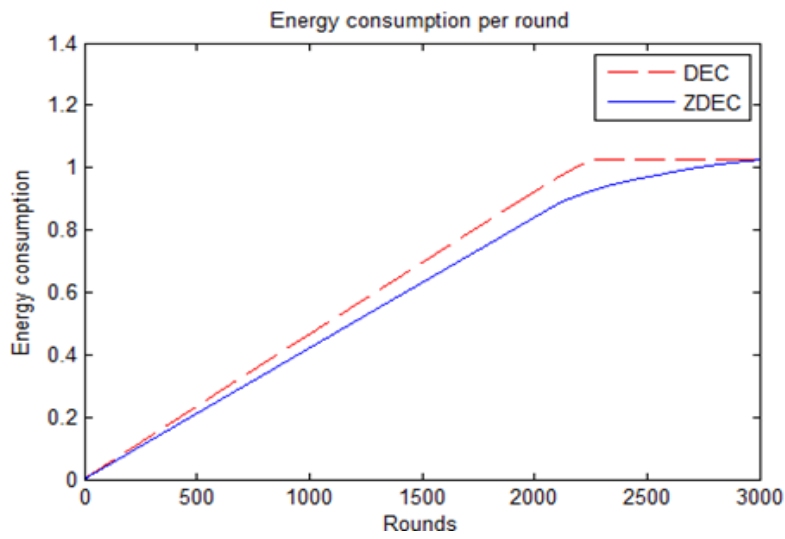

Fig 8: Energy consumption per round

The analysis of number of cluster heads elected per round is given in Fig. 9. 


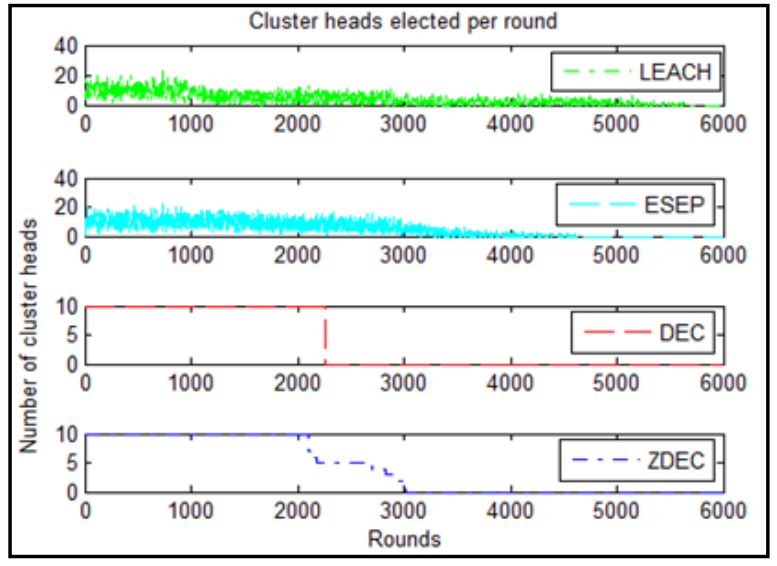

Fig 9: Number of cluster heads elected per round

It is depicted from the figure that the LEACH and ESEP shows variation in electing cluster heads with respect to round and hence their energy dissipation is also not uniform. The uncertainties in election of cluster head have been almost eradicated in ZDEC and DEC protocol. Once the required optimal number of cluster heads to be elected in each round is decided in the beginning, our proposed ZDEC protocol ensures the election of those required number of cluster-heads in each round in any network size till the stability period. Thus most of the time cluster-members have to transmit data to their cluster-heads relatively at shorter distances. It leads to the uniform energy dissipation over the sensor field. Overall, the energy gain by ZDEC is reflected in simulation results in its favor.

\section{CONCLUSION}

In this paper, we have proposed ZDEC, a zonal based clustering protocol in which field is partitioned into zones. Cluster heads are elected on the basis of residual energy such that each zone contains minimum one cluster heads. Doing such kind of partitioning and election of cluster head in each zone ensures uniform and well distributed election of $\mathrm{CHs}$ throughout layout of network. Hence it yields efficient energy utilization of sensor nodes. Simulations show that ZDEC has more stability period than LEACH, ESEP and DEC protocols. It is best suited for those critical applications that require $90 \%$ to $100 \%$ monitoring and hence to get reliable data for long period of time.

\section{REFERENCES}

[1] Aderohunmu, F.A. and Deng, J.D. 2009. An Enhanced Stable Election Protocol (SEP) for Clustered Heterogeneous WSN.

[2] Aderohunmu, F.A., Deng, J.D. and Purvis, M.K.. 2011. A deterministic energy-efficient clustering protocol for wireless sensor networks. In Proceedings of Seventh IEEE International Conference on Intelligent Sensors, Sensor Networks and Information Processing, pp.341 346.

[3] Heinzelman, W.R., Chandrakasan, A. and Balakrishnan, H. 2000. Energy-efficient communication protocol for wireless microsensor Networks. In Proceedings of the 33rd Hawaii International Conference on System Sciences (HICSS-33).

[4] Li, C.F., Ye, M. and Chen, G.H. 2005. An energyefficient unequal clustering mechanism for wireless sensor networks," In Proceedings of the 2nd IEEE International Conference on Mobile Adhoc and Sensor Systems (MASS 2005), Washington, DC , pp. 597-604.

[5] Manjeswar, A. and Agrawal, D.P. 2001. TEEN: A protocol for enhanced efficiency in wireless sensor networks. In Proceedings of 1st International Workshop on Parallel and Distributed Computing Issues in Wireless Networks and Mobile Computing, San Francisco, CA, USA.

[6] Smaragdakis, G., Matta, I. and Bestavros, A. 2004. A Stable Election Protocol for clustered heterogeneous wireless sensor networks. In Proceeding of the International Workshop on SANPA.

[7] Kaur, P. and Singh, R. 2014. J. 2000. DDEC: Distance based deterministic energy efficient clustering protocol for wireless sensor networks. In Proceedings of International Conference on Advances in Computing, Communications and Informatics (ICACCI). 